\title{
Psychoses and their treatments: a dual threat for the patients' total health? Radjiv Tandon*
}

Address: University of Florida, USA

* Corresponding author

from International Society on Brain and Behaviour: 2nd International Congress on Brain and Behaviour Thessaloniki, Greece. 17-20 November 2005

Published: 28 February 2006

Annals of General Psychiatry 2006, 5(Suppl I):S25 doi:I0.II86/I744-859X-5-SI-S25

The primary objective of treating any illness is to minimize the effects of the illness on the patient's life, thereby enabling the individual to lead the highest achievable quality of life. Schizophrenia is a chronic disorder with devastating effects on several aspects of the patient's life and is associated with a shortened lifespan (age-standardized mortality twice that of the general population), a high risk of suicide (5-10\% of patients die from suicide) and significant impairment in function (only about $10 \%$ of patients are fully employed and live independently). The broad objective of treatment is to reduce the overall morbidity and mortality of the disorder by decreasing the frequency and severity of episodes of psychotic exacerbation and improving the functional capacity and quality of lives of the individuals afflicted with the illness. Whereas antipsychotic medications are useful in reducing many manifestations of the illness, their adverse effects can adversely impact the morbidity and mortality of the illness. Whereas second-generation antipsychotics demonstrate a broader spectrum of efficacy than first-generation agents and have a lower propensity to cause neurological side-effects, they are associated with varying degrees of metabolic side-effects that substantially increase the risk of ischemic heart disease (the single-largest cause of death in schizophrenia). While available second-generation antipsychotics are broadly similar in terms of their efficacy, they vary significantly with regards to their sideeffect profiles. In this presentation, a model of conceptualizing the impact of treatment on the lives of individuals suffering from schizophrenia will be presented and antipsychotic treatment approaches that maximize effectiveness for each individual patient summarized. 